\title{
Flexible and efficient model-based congestion detection approach
}

\author{
Zeroual Abdelhafid \\ LAIG Laboratory, University of 08 May 1945, \\ Guelma 24000, Algeria \\ CReSTIC, university of Reims \\ Champagne-ardenne, France \\ Email: ab_zeroual@yahoo.com
}

\author{
Fouzi Harrou, Member, IEEE, Ying Sun \\ King Abdullah University of Science and Technology (KAUST), \\ Computer, Electrical and Mathematical \\ Sciences and Engineering (CEMSE) Division, \\ Thuwal, 23955-6900, Saudi Arabia, Email: fouzi.harrou@kaust.edu.sa
}

\begin{abstract}
This paper addresses the problem of road traffic congestion detection. We propose an effective approach to detect traffic congestion by combining the piecewise switched linear traffic (PWSL) and Shewhart control scheme. This approach uses PWSL model to describe the evolution of traffic density, and Shewhart chart to detect traffic congestions based on residuals obtained from PWSL model. The PWSL-Shewhart approach is evaluated using traffic data from the four-lane State Route 60 (SR-60) freeway in California. Results indicate that our approach accomplished reliable detection of traffic congestion.
\end{abstract}

Keywords-Traffic density, macroscopic modelling, CTM, PWSL model, monitoring chart.

\section{INTRODUCTION}

Intelligent transportation systems becomes a vital element in the development of our society [1]. Road traffic congestion is among the most growing areas of concern for many countries [2], [3]. Traffic congestion management and monitoring in public road networks and highways are becoming increasingly important factors for economic growth of countries [4]. Accordingly, reliable detection of traffic congestion plays a key role in improving traffic safety and comfort of driving [5], [6]. In this paper, the focus will be on the detection of traffic congestion using a statistical method.

Flexible and accurate modeling of road traffic is important to enhance road traffic monitoring. Recently, there has been much discussion on road traffic modeling in the literature [4] [6]. Microscopic and macroscopic approaches are two main categories in traffic modeling framework [7]-[9]. Microscopic models are mainly focusing on the individual comportment of vehicles. While macroscopic models are based on the hydrodynamic theory for capturing the traffic dynamics as continues flow. Generally speaking, macroscopic models use a reduced number of parameters compared to microscopic approaches, which lead to less computational complexity and time consuming [7]. This paper is within the macroscopic modeling framework. Specifically, we focus on the cell transmission model (CTM), which is a discretized version of the first order macroscopic model [8]. Here, the piecewise switched linear model (PWSL), which is an enhanced version of CTM [9] will be used as a modeling framework. The main benefit of the PWSL model is its capability and flexibility to be modular for several configurations of road portions with various numbers of cells and junctions.

With the increasing needs for traffic monitoring and supervision, various techniques aimed at detecting road traffic congestion have been proposed including multi-layer feed forward neural network [10], Markov model and back propagation neural network [11], probability neural network, constructive probability neural network [12], [13] and support vector machine [14]. These machine learning methods require high computational training time, which makes them less attractive for online application. Many other interesting approaches for traffic monitoring were discussed in the literature, for a more complete discussion on traffic modeling and monitoring approaches see [4]-[6]. While several fault detection techniques have been proposed for traffic monitoring [15], [16], statistical monitoring schemes have not been widely exploited for monitoring of traffic congestion until recently [17]-[19].

In this paper, an efficient detection strategy to monitor traffic congestion is presented. This strategy combines the benefits of both the PWSL modeling and Shewhart monitoring chart. In fact, the PWSL model is used to capture traffic dynamics and describe traffic flow evolution. Then, we verify the residuals the PWSL model using the Shewhart scheme for checking the traffic state (i.e., congested or free traffic). We applied the proposed PWSL-Shewhart approach to real traffic data from a portion of the SR60-E highway in California. Results show the capability of this approach to reveal the presence of road traffic congestion.

The following section briefly reviews the macroscopic traffic modeling and the improved piecewise macroscopic traffic model. Section III briefly describe the proposed PWSLShewhart approach. In Section IV, the performances of the proposed method are illustrated through real data. Finally, Section V concludes the paper.

\section{Piecewise Switched Linear Macroscopic TRAFFIC MODEL}

The cell transmission model (CTM) is one of the most used traffic models in the literature [8]. It is a first order discretized 
macroscopic traffic model, which considers the road section as successive segments or cells of length $\Delta x_{i}$ (Fig. 1).

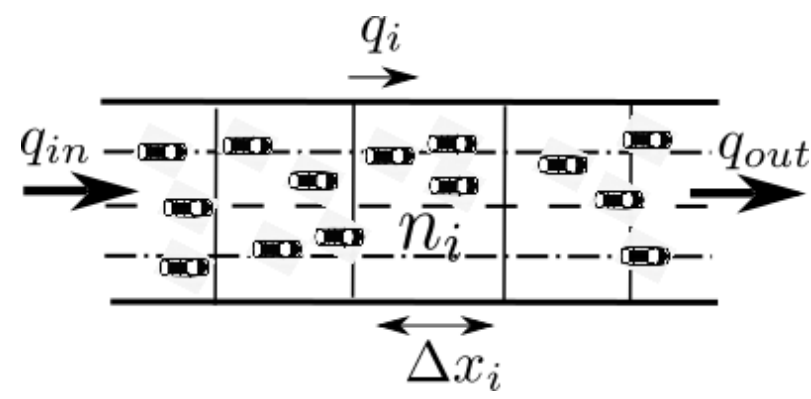

Fig. 1. Illustration of the spatial road segmentation.

The dynamics of vehicle numbers in each cell $i$ (occupancy) is expressed by:

$$
n_{i}(k+1)=n_{i}(k)+\Delta T\left(q_{i}(k)-q_{i+1}(k)\right),
$$

where, the flow $q_{i}(k)$ in each cell $i$ is obtained as follows:

$$
q_{i}(k)=\min \left(n_{i-1}(k), Q_{M, i}, \frac{w_{i}}{v_{f, i}}\left(n_{j, i}-n_{i}(k)\right)\right),
$$

where $n_{j, i}$ is the capacity of the cell $i$.

The macroscopic model's parameters can be represented by the fundamental diagram FD (Figure 2), which is the empirical curve of the relationship flow-density. These parameters are: free flow speed $v_{f, i}$, critical density $\rho_{c, i}$, maximal flow, congested wave speed $w_{i}$ and jam density $\rho_{j, i}$ (see Figure 2).

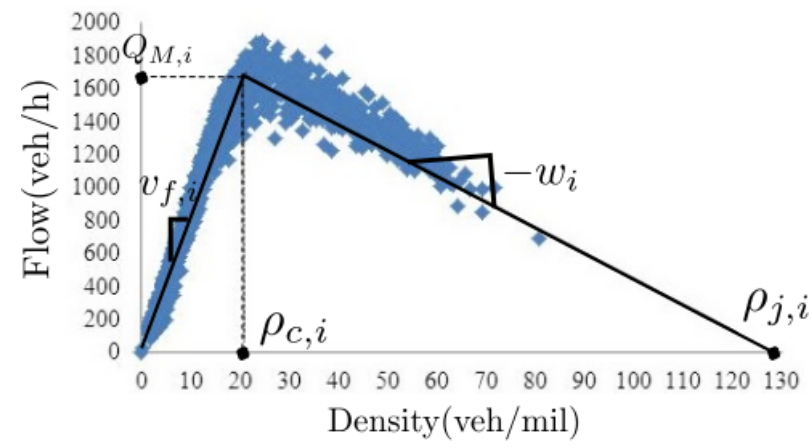

Fig. 2. Illustration of fundamental diagram triangular form.

To solve the CTM nonlinearity and for more realistic representation of traffic density, an extended and enhanced version called the Piecewise Switched Linear (PWSL) model is introduced in [9]. It is a hybrid dynamic system of $2 \times N$ normal modes that obtained by the piecewise linearization of the CTM model according to the both of upstream and downstream directions of wave congestion propagation. The general representation of the PWSL is given as:

$$
\begin{gathered}
\rho(k+1)=A_{s} \rho(k)+B_{s} u(k)+D_{s} \\
D_{s}=B_{j a m, s} \rho_{j}+B_{Q, s} Q_{M} \\
y(k)=c \rho(k)
\end{gathered}
$$

where $s=\{1, \ldots, 2 N\}$ indicates the mode of the system, $\rho(k)=\left[\rho_{1}(k), \ldots, \rho_{N}(k)\right]^{T}$ is the density state vector, $u(k)=\left[q_{\text {in }}(k), q_{O N, 2}(k), \ldots, q_{O N, N-1}(k), q_{\text {out }}(k)\right]^{T}$ is the entering flow vector (input, output and on-ramp flow), $Q_{M}=\left[Q_{M, 1}, \ldots, Q_{M, N}\right]^{T}$ is the maximal flow vector, $\rho_{j}=$ $\left[\rho_{j, 1}, \ldots, \rho_{j, N}\right]$ the jam density vector, $y(k)$ is the system output and $\left(\rho(k), u(k), Q_{M}, \rho_{j}\right) \in \mathbb{R}^{N \times 1}, A_{s} \in \mathbb{R}^{N \times N}, B_{s}$ $\in \mathbb{R}^{N \times N}, D_{s} \in \mathbb{R}^{N \times N}$ and $c \in \mathbb{R}^{2 \times N}$ are known matrices. For more details about the PWSL model see [9].

\section{PWSL-SHEWHART CONGESTION MONITORING MECHANISM}

To develop an efficient congestion detection procedure, we have mixed the PWSL model with Shewhart scheme. Shewhart chart (termed also three-sigma rule) has been proposed by Walter Shewhart [20]. Shewhart charts are suitable in detecting large mean shifts [21], [22].

In a Shewhart chart, a sequence of samples $x_{i}$ are plotted against time [20]. Upper and lower control limits (UCL, LCL), for the samples are established as following:

$$
U C L, L C L=\mu_{0} \pm 3 \sigma_{0},
$$

where $\mu_{0}$ is the process mean and $\sigma_{0}$ is the standard deviation of the congestion-free data. If PWLS residuals are outside the control limits, a signal of congestion is flagged.

In the proposed procedure, we applied Shewhart chart to PWSL residuals, $e$, which are the difference between the measured $(\rho)$ and the predicted $(\hat{\rho})$ densities, to detect possible traffic congestions (see Figure 3). PWSL residuals are close to zero in normal traffic and deviate significantly from zero under congested situations. When the PWSL residuals overpass the control limits of the Shewhart chart, we declare an abnormal traffic situation.

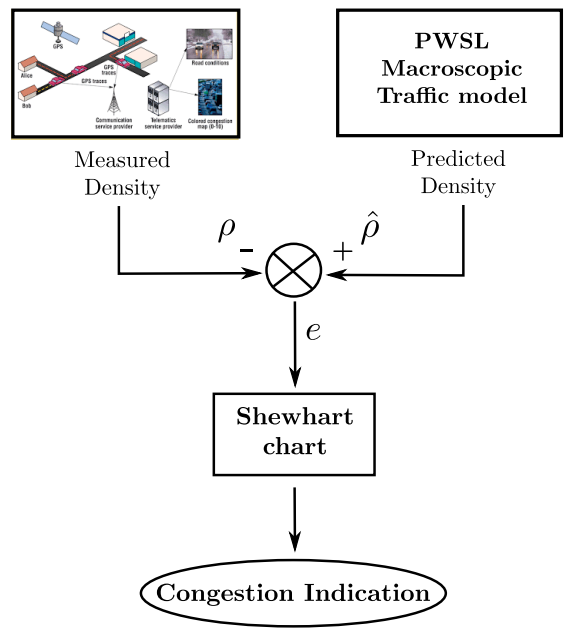

Fig. 3. Illustration of PWSL-Shewhart monitoring mechanism. 


\section{Simulation RESUlts}

\section{A. Data description}

To verify the performance of the proposed congestion detection procedure, we consider data from a highway portion of 1.73 miles stretch of SR60, which is a four-lane highway from the east of California (Fig. 4). Respecting the CourantFriedrichs-Lewy conditions $\left(\Delta T v_{f, i} \leq \Delta x_{i}\right)$, the selected portion has been partitioned into four cells. Each cell has equipped with a vehicle detector stations (VDS), which provides traffic data.

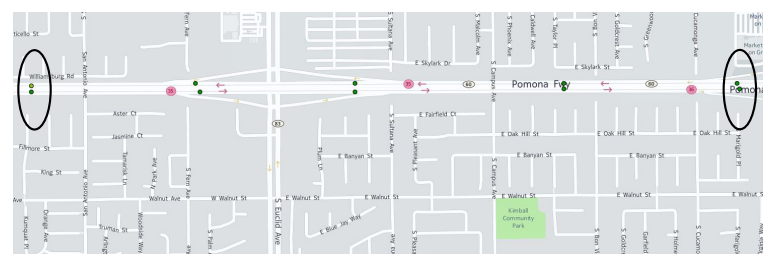

(a) Road map SR60-E highway portion

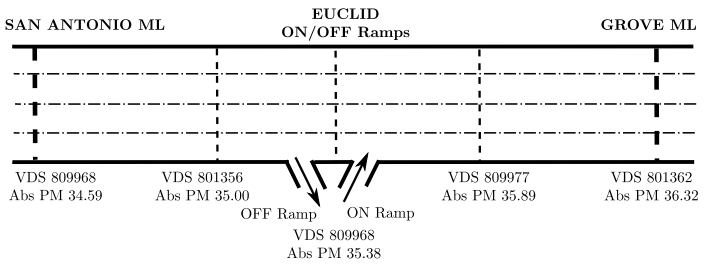

Abs PM: Absolute Post Mile VDS: Vehicle Detector Station

(b) Schematic representation of the SR60-E portion

Fig. 4. The studied SR60-W highway portion.

Data calibration or fundamental diagram identification is a necessary step for each macroscopic model validation [23]. In which, we applied the calibration methodology proposed in [9], [24] on the considered road portion. Then, we identified the triangular fundamental diagram form for each cell and we estimated the PWSL model parameters for each cell.

\section{B. Model validation}

The constructed PWSL model is fitted to the free flow data training dataset from four different cells, and the goodness of fit is shown in Figure 5(a)-(d). From Figure 5, we can see that the four time series (cell 01, cell 02, cell 03 and cell 04) are well-adjusted by the constructed models.

Furthermore, to illustrate the quality of the PWSL-based models selected, the scatter plot of measured vehicle density data versus predicted values of the constructed PWSL models are presented in Figure 6. According to such a figure, it is clear that the measured data are well-fitted by the PWSL model.

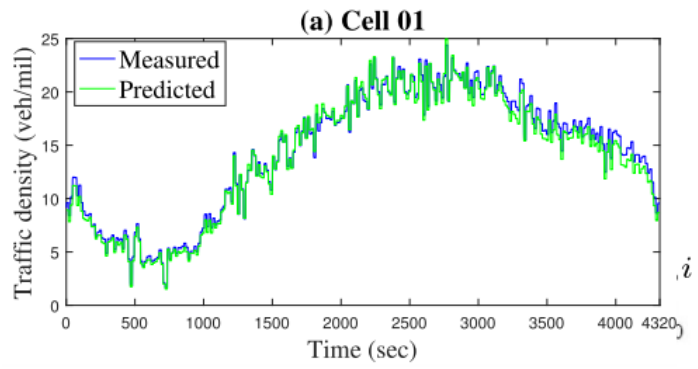

(b) Cell 02

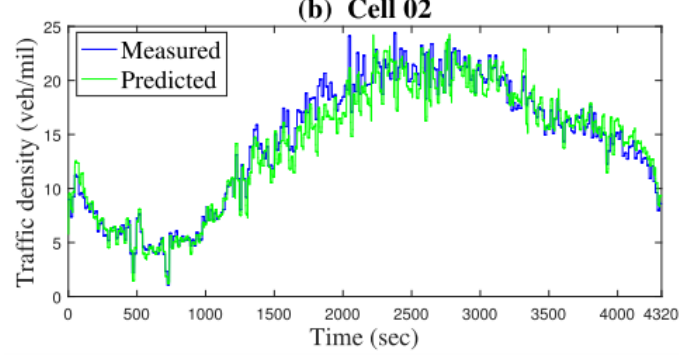

(c) Cell 03

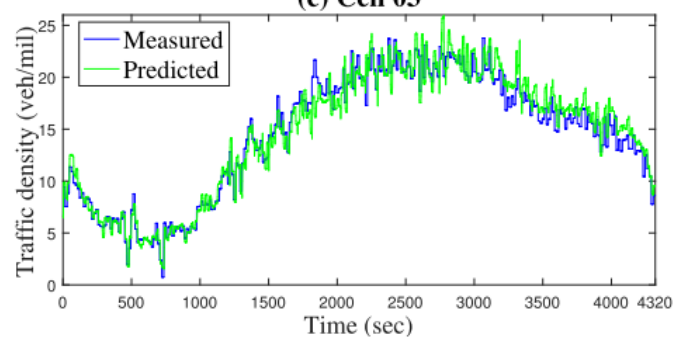

(d) Cell 04

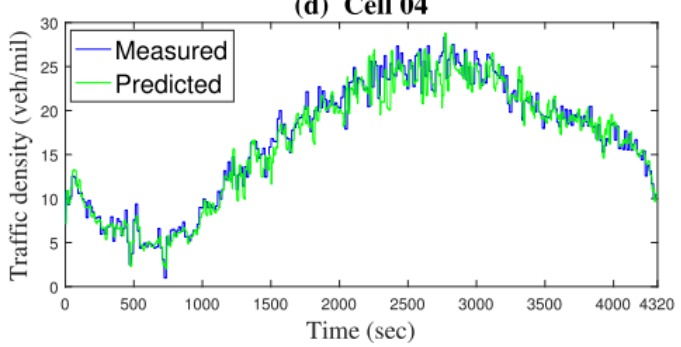

Fig. 5. Measured and PWSL predicted traffic density from SR60 dataset, June 23, 2012.
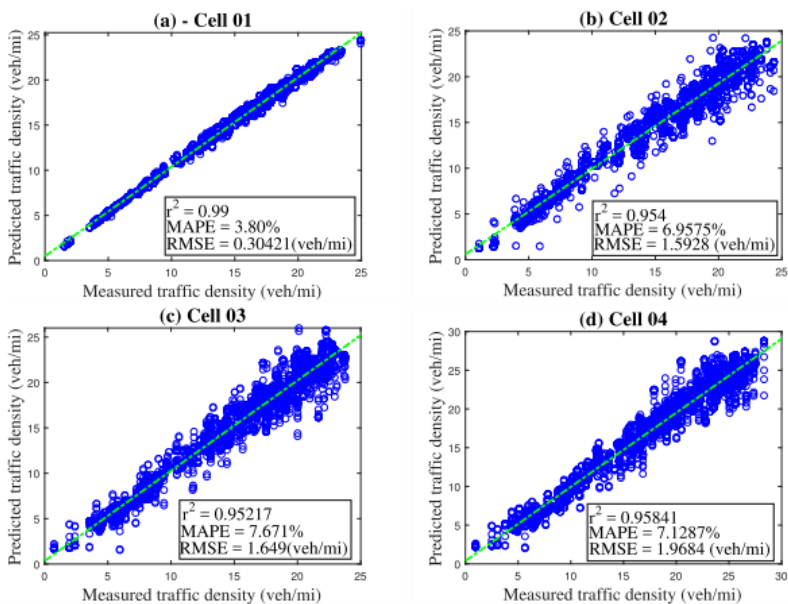

Fig. 6. Scatter plots of the measured and the PWSL predicted traffic density from SR60 dataset. 
To further evaluate the accuracy of the constructed PWSL model, three numerical criteria were employed: $R^{2}$, the mean absolute percent error (MAPE) and the root mean square error (RMSE) (Table I). Table I shows that the PWSL model describes appropriately the traffic density.

TABLE I

QUALITY OF THE PWSL MODEL WHEN APPLIED TO DATA FROM FIGURE 5.

\begin{tabular}{|c|c|c|c|c|}
\hline & Cell 01 & Cell 02 & Cell 03 & Cell 04 \\
\hline$r^{2}$ & 0.99 & 0.954 & 0.9522 & 0.9584 \\
\hline RMSE & 0.3042 & 1.5928 & 1.649 & 7.1287 \\
\hline MAPE & 3.80 & 6.9575 & 7.671 & 1.9684 \\
\hline
\end{tabular}

\section{Detection results}

To assess the performance of PWSL-Shewhart approach, three case studies are presented here.

1) Scenario with abrupt congestions: Here, we evaluate the proposed approach in the case of road traffic with abrupt congestion. The abrupt congestion is simulated by adding a small constant deviation between samples 1600 and 1800 . The magnitude of the deviation is equal to $10 \%$ of the total variation in the raw data. This could represent an incident such as stalled cars, road repairs, overturned vehicles, and bad weather. Results of PWSL-Shewhart approach based on unfiltered are desplayed in Figure 7. Figure 7 shows that the PWSL-Shewhart is able to detect the congestion, but with several false alarms.

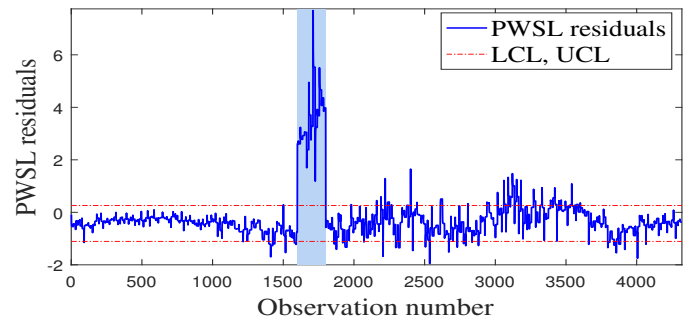

Fig. 7. PWSL-Shewhart procedure with the unfiltered residuals, scenario with an abrupt congestion.

The performance of PWSL-Shewhart approach can be improved by using filtered residuals via the wavelet-based filter (Figure 8). We can see from Figure 8 that the number of false alarms is reduced by using the filtered residuals.

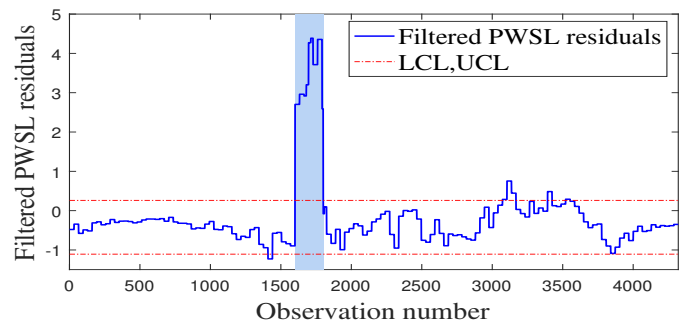

Fig. 8. PWSL-Shewhart procedure with the filtered residuals, scenario with an abrupt congestion.
2) Scenario with intermittent congestions: In the second scenario, we inject into the testing data a bias of amplitude $3 \%$ of the total variation in the testing data between samples 1600 and 1800, and a bias of $10 \%$ from sample 3000 to sample 3500. The monitoring results of the Shewhart chart based on unfiltered residuals are demonstrated in Figure 9. From Figure 9, it can be seen that the Shewhart chart is capable of detecting these moderate abnormal congestion but with some missed detections. Figure 10 shows that PWSLShewhart approach provides better performance when it is applied to filtered residuals.

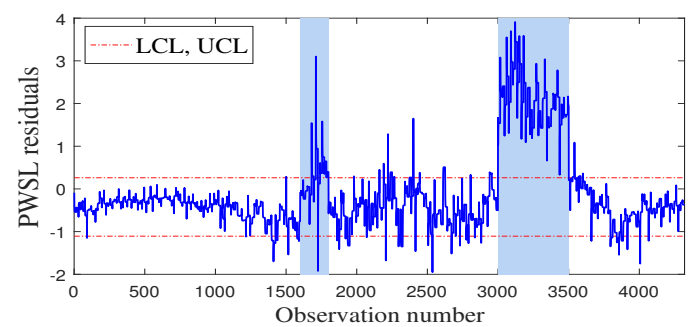

Fig. 9. PWSL-Shewhart procedure with the unfiltered residuals, scenario with an intermittent congestion.

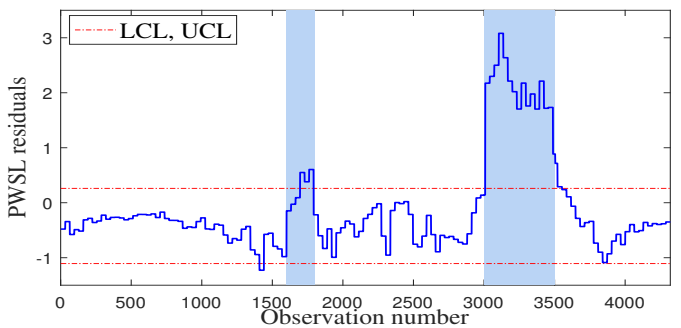

Fig. 10. PWSL-Shewhart chart with the smoothed residuals, scenario with an intermittent congestion.

3) Scenario with gradual congestions: The aim of the third scenario is to evaluate the potential of the proposed PWSLShewhart chart to detect gradual congestion. Here, a slow increase with a slope of 0.01 was added to the simulated test data starting at sample number 3000 . Figure 11 shows that the Shewhart chart statistic, when applied to the unfiltered data, exceeds the control limit after the occurrence of congestion at sample number 3042, but with several false alarms.

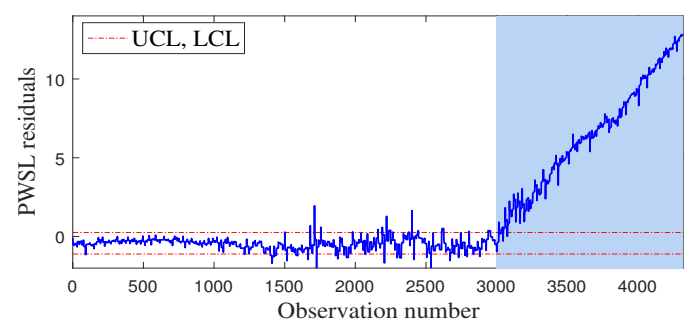

Fig. 11. PWSL-Shewhart procedure with the unfiltered residuals, scenario with a gradual congestion. 
The Shewhart chart when applied to the smoothed residuals detect this gradual congestion with fewer false alarms (Figure 12). These results also show that applying the proposed approach on pre-filtered residuals slightly enhanced its detection ability by reducing the number of false alarms.

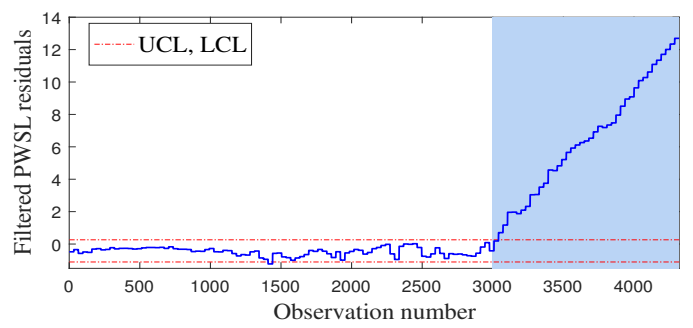

Fig. 12. PWSL-Shewhart procedure with the smoothed residuals, scenario with a gradual congestion.

\section{CONCLUSION}

This paper introduces a flexible statistical procedure for supervising traffic congestions. We used PWSL model to describe the traffic density dynamics, and Shewhart control chart to detect abnormal traffic congestions. Indeed, Shewhart chart is applied to the residuals obtained from the PWSL model to take a decision about road traffic state (i.e., congested or free traffic). We validated the PWSL-Shewhart approach via real data from SR-60 California highway. Results show the ability of the PWSL-Shewhart approach in detecting road traffic congestion.

\section{ACKNOWLEDGEMENT}

This publication is based upon work supported by the King Abdullah University of Science and Technology (KAUST) Office of Sponsored Research (OSR) under Award No: OSR2015-CRG4-2582.

\section{REFERENCES}

[1] X. Cheng, L. Yang, and X. Shen, "D2d for intelligent transportation systems: A feasibility study," IEEE Transactions on Intelligent Transportation Systems, vol. 16, no. 4, pp. 1784-1793, 2015.

[2] J. Barria and S. Thajchayapong, "Detection and classification of traffic anomalies using microscopic traffic variables," IEEE Transactions on Intelligent Transportation Systems, vol. 12, no. 3, pp. 695-704, 2011.

[3] B. Williams and A. Guin, "Traffic management center use of incident detection algorithms: Findings of a nationwide survey," IEEE Transactions on intelligent transportation systems, vol. 8, no. 2, pp. 351-358, 2007.

[4] L. Adacher and M. Tiriolo, "A macroscopic model with the advantages of microscopic model: A review of cell transmission modelâÁŹs extensions for urban traffic networks," Simulation Modelling Practice and Theory, vol. 86, no. August, pp. 102-119, 2018.

[5] A. Zeroual, F. Harrou, Y. Sun, and N. Messai, "Integrating model-based observer and kullback-leibler metric for estimating and detecting road traffic congestion," IEEE Sensors Journal, pp. 1-11, 2018.

[6] T. Seo, A. M. Bayen, T. Kusakabe, and Y. Asakura, "Traffic state estimation on highway: A comprehensive survey," Annual Reviews in Control, vol. 43, pp. 128-151, 2017.

[7] A. Gning, L. Mihaylova, and R. K. Boel, "Interval Macroscopic Models for Traffic Networks," IEEE Transactions on Intelligent Transportation Systems, vol. 12, no. 2, pp. 523-536, jun 2011.
[8] C. F. Daganzo, "The cell transmission model: A dynamic representation of highway traffic consistent with the hydrodynamic theory," Transportation Research Part B: Methodological, vol. 28, no. 4, pp. 269-287, aug 1994.

[9] A. Zeroual, N. Messai, S. Kechida, and F. Hamdi, "A Piecewise Switched Linear Approach for Traffic Flow Modeling," International Journal of Automation and Computing, 2017.

[10] E. Chang, "A neural network approach to freeway incident detection," in The 3rd International Conference on Vehicle Navigation and Information Systems, VNIS. IEEE, 1992, pp. 641-647.

[11] X. Yu, S. Xiong, Y. He, W. E. Wong, and Y. Zhao, "Research on campus traffic congestion detection using bp neural network and markov model," Journal of Information Security and Applications, vol. 31, pp. 54-60, 2016.

[12] S. Ritchie and B. Abdulhai, "Development testing and evaluation of advanced techniques for freeway incident detection," California Partners for Advanced Transit and Highways (PATH), 1997.

[13] X. Jin, D. Srinivasan, and R. Cheu, "Classification of freeway traffic patterns for incident detection using constructive probabilistic neural networks," IEEE Transactions on Neural networks, vol. 12, no. 5, pp. 1173-1187, 2001.

[14] R. Cheu, D. Srinivasan, and E. Teh, "Support vector machine models for freeway incident detection," in Proceedings on Intelligent Transportation Systems, vol. 1. IEEE, 2003, pp. 238-243.

[15] E. D'Andrea and F. Marcelloni, "Detection of traffic congestion and incidents from gps trace analysis," Expert Systems with Applications, vol. 73, no. 2017, pp. 43-56, 2017.

[16] A. Kinoshita, A. Takasu, and J. Adachi, "Real-time traffic incident detection using a probabilistic topic model," Information Systems, vol. 54, pp. 169-188, 2015.

[17] A. Zeroual, F. Harrou, Y. Sun, and N. Messai, "Monitoring road traffic congestion using a macroscopic traffic model and a statistical monitoring scheme," Sustainable Cities and Society, vol. 35, no. Supplement C, pp. $494-510,2017$

[18] A. Zeroual, F. Harrou, and y. Sun, "An efficient statistical-based approach for road traffic congestion monitoring," in The 5th International Conference on Electrical Engineering ICEE2017B. IEEE, 2017, pp. $1-5$.

[19] F. Harrou, A. Zeroual, and Y. Sun, "Traffic congestion detection based on hybrid observer and GLR test," in 2018 Annual American Control Conference (ACC). IEEE, 2018, pp. 604-609.

[20] D. C. Montgomery, "Introduction to statistical quality control," John Wiley\& Sons, New York, 2005.

[21] E. Garoudja, F. Harrou, Y. Sun, K. Kara, A. Chouder, and S. Silvestre, "Statistical fault detection in photovoltaic systems," Solar Energy, vol. 150, no. Supplement C, pp. 485 - 499, 2017.

[22] F. Kadri, F. Harrou, S. Chaabane, Y. Sun, and C. Tahon, "Seasonal arma-based spc charts for anomaly detection: Application to emergency department systems," Neurocomputing, vol. 173, pp. 2102-2114, 2016.

[23] G. Gomes and R. Horowitz, "Automatic Calibration of the Fundamental Diagram and Empirical Observations on Capacity," Transportation Research Board, no. January, pp. 1-14, 2009.

[24] A. Zeroual, N. Messai, S. Kechida, and F. Hamdi, "Calibration and Validation of a Switched Linear Macroscopic Traffic Model," in 3rd International Conference on Control, Engineering \& Information Technology CEIT2015, no. 1. IEEE, 2015, pp. 25-27. 